\title{
Méthodologie pour une utilisation conjointe de données eulériennes et langrangiennes de courant pour estimer un coefficient de diffusion horizontale côtières Methodology for Joint Use of Eulerian and Lagrangian Measurements in Order to Estimate Horizontal Diffusion Coefficients in Coastal Zones
}

\author{
N. Baghdadi et P. Broche
}

Volume 8, numéro 3, 1995

URI : https://id.erudit.org/iderudit/705231ar

DOI : https://doi.org/10.7202/705231ar

Aller au sommaire du numéro

\section{Éditeur(s)}

Université du Québec - INRS-Eau, Terre et Environnement (INRS-ETE)

ISSN

0992-7158 (imprimé)

1718-8598 (numérique)

Découvrir la revue

Citer cet article

Baghdadi, N. \& Broche, P. (1995). Méthodologie pour une utilisation conjointe de données eulériennes et langrangiennes de courant pour estimer un coefficient de diffusion horizontale côtières. Revue des sciences de l'eau/ Journal of Water Science, 8(3), 403-421. https://doi.org/10.7202/705231ar

\section{Résumé de l'article}

Le Laboratoire de Sondages Electromagnétiques de l'Environnement Terrestre a une pratique confirmée de la mise en oeuvre des radars Doppler VHF pour la réalisation de mesures eulériennes des courants marins en zone côtière. Avec ce même instrument, on peut réaliser, simultanément aux mesures eulériennes, des mesures lagrangiennes en effectuant le suivi, avec le radar, de flotteurs dérivants de surface équipés de balises.

Nous proposons une méthode d'utilisation couplée des données eulériennes et lagrangiennes pour estimer l'importance des phénomènes de diffusion horizontale. L'influence de la résolution spatiale des mesures de courant et du nombre de flotteurs sur la précision des mesures de dispersion a été discutée. Une validation de cette méthode a été effectuée à partir d'une étude numérique sur un exemple de panache fluvial (I'étude de ce dernier est un des axes principaux de recherche du LSEET). Cette étude montre que l'erreur sur l'estimation de ce coefficient pour 5 bouées lâchées et avec une résolution spatiale de (500 x 500) m- peut être de l'ordre de 35\%. On obtient ainsi un instrument particulièrement complet de diagnostic dynamique en zone côtière, présentant un grand intérêt pour l'étude d'un rejet ou le transport d'un contaminant. 


\title{
Méthodologie pour une utilisation conjointe de données eulériennes et lagrangiennes de courant pour estimer un coefficient de diffusion horizontale en zones côtières
}

\author{
Methodology for joint use of Eulerian and Lagrangian \\ measurements in order to estimate horizontal \\ diffusion coefficients in coastal zones
}

N. BAGHDADI et P. BROCHE

Reçu le 19 janvier 1995, accepté le 7 juin 1995*.

SUMMARY

VHF Doppler radars have been used by LSEET for the measurements of Eulerian superficial currents in coastal zones (between 1 to $30 \mathrm{~km}$ from the shore). For radar frequencies close to $50 \mathrm{MHz}$, the velocity measured is integrated over a depth of $25 \mathrm{~cm}$, over an area of a few hundred square metres, and for times of one to ten minutes. The accuracy of the velocity measurements is better than 5 $\mathrm{cm} / \mathrm{s}$. The radars are set up on the sea-shore and their maximum range, depending upon sea-state, is of $20-30 \mathrm{~km}$. Two radars are needed to obtain a mapping of current vectors.

These measurements don't account for velocity fluctuations at scales less than the mesh size, which are generally described by a diffusion coefficient $\left(K_{h}\right)$.

On the other hand, Lagrangian measurements can be made by tracking surface drifters. The same VHF radars can be used for such tracking by using radio beacons placed on the drifters.

This paper proposes a method for using both the Eulerian velocity field (usual product of VHF radars) and the Lagrangian trajectories of several drifters in order to estimate the horizontal diffusion coefficient. This method consists of comparing the statistical characteristics of the observed drifters distribution with those obtained by numerical simulations based on a random walk method. Numerical experiments concerning the influence of the accuracy and the spatial resolution of current measurements and the effect of the number of drifters on the accuracy of dispersion estimate have been carried out. In the particular case of a river plume with an outflow of the order of $1000 \mathrm{~m} 3 / \mathrm{s}$, we have shown that the accuracy of the horizontal diffusion coefficient estimation is of the order of $35 \%$ for the realistic number of 5 drifters, with a spatial resolution of $500 \times 500 \mathrm{~m}^{2}$.

1. Laboratoire de Sondages Electromagnétiques de l'Environnement Terrestre LSEET-CNRS/Université de Toulon et du Var BP 132 - B3957 La Garde Cedex France

* Les commentaires seront rę̧us jusqu'au 2 mai 1996. 
The system (radars and drifters) provides a powerful tool of a dynamic diagnosis in the coastal zone, and should be of interest for the study of discharges or the evolution of waterborne pollutants.

Key-words : VHF radars, drifters, Eulerian and Lagrangian currents, numerical simulation, random walk, diffusion coefficient.

\section{RÉSUMÉ}

Le Laboratoire de Sondages Electromagnétiques de l'Environnement Terrestre a une pratique confirmée de la mise en cuure des radars Doppler VHF pour la réalisation dê mesüures eulériennes des courants marins en zone côtière. Avec ce même instrument, on peut réaliser, simultanément aux mesures eulériennes, des mesures lagrangiennes en effectuant le suivi, avec le radar, de flotteurs dérivants de surface équipés de balises.

Nous proposons une méthode d'utilisation couplée des données eulériennes et lagrangiennes pour estimer l'importance des phénomènes de diffusion horizontale. L'influence de la résolution spatiale des mesures de courant et du nombre de flotteurs sur la précision des mesures de dispersion a été discutée. Une validation de cette méthode a été effecturée à partir d'une étude numérique sur un exemple de panache fluvial (I'étude de ce dernier est un des axes principaux de recherche du LSEET). Cette étude montre que l'erreur sur l'estimation de ce coefficient pour 5 bouées lâchées et avec une résolution spatiale de $(500 \times 500)$ $\mathrm{m}^{2}$ peut être de l'ordre de $35 \%$. On obtient ainsi un instrument particulièrement complet de diagnostic dynamique en zone côtière, présentant un grand intérêt pour l'étude d'un rejet ou le transport d'un contaminant.

Mots clés : radars VHF, bouées dérivantes de surface, courantométrie eulérienne et lagrangienne, simulation numérique, marche au hasard, coefficient de diffusion.

\section{1 - INTRODUCTION}

Les radars VHF sont des instruments de télédétection et de poursuite avec lesquels il est possible de réaliser simultanément des mesures eulériennes et lagrangiennes de courant en zone côtière. Les radars en fonctionnement classique comme courantomètres foumissent une bonne estimation du champ de courant sur une grille spatio-temporelle discrète de dimension $30 \times 30 \mathrm{~km}^{2}$ (fig. 1); les mailles de cette grille sont de l'ordre de $500 \times 500 \mathrm{~m}^{2}$. Les courants eulériens, mesurés avec une précision meilleure que $5 \mathrm{~cm} / \mathrm{s}$, sont intégrés spatialement sur les mailles de la grille (BROCHE et al., 1987 ; DEVENON et al., 1991), sur une profondeur d'une vingtaine de centimètres (STEWART et JOY, 1974) et sur une durée de une à quelques minutes. Les radars sont installés sur la côte, et la portée, variable avec l'état de la mer, est de 20 à $30 \mathrm{~km}$.

La réalisation des mesures lagrangiennes de courant peut être effectuée grâce à un suivi, par le radar, de bouées dérivantes de surface. Les bouées 
dérivantes sont de petites dimensions, de faible poids (inférieur à $10 \mathrm{~kg}$ ) et équipées de balises pour permettre leur localisation par radar. Elle sont conçues pour suive les masses d'eau à une profondeur de $25 \mathrm{~cm}$. La portée actuelle d'une balise est d'environ $20 \mathrm{~km}$ par « petite " mer et sa localisation par le radar est effectuée à mieux que $100 \mathrm{~m}$ (BAGHDADI et al., 1994). On peut donc estimer que la précision du positionnement est suffisante pour effectuer des mesures de courants de dérive.

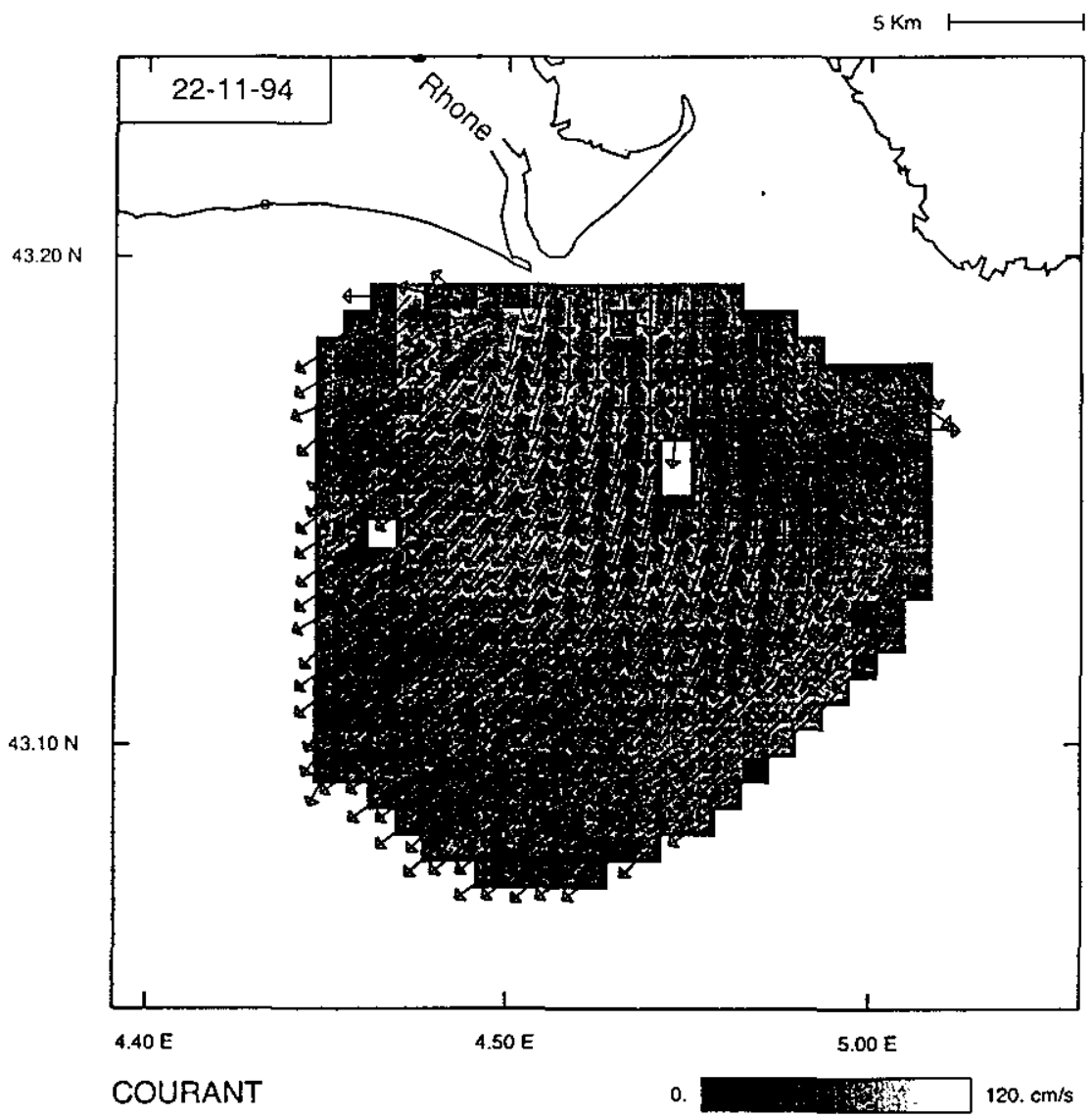

Figure 1 Exemple de résultats de mesures eulériennes de courant sur le panache du Rhône (1994). Les valeurs représentées sont celles du vecteur courant.

Current vectors on the sea surface as mapped by VHF Doppler radars in 1994 off Rhône river mouth.

Dans le présent article, nous proposons une méthode d'utilisation couplée des données eulériennes et lagrangiennes de courant pour estimer l'importance des phénomènes de dispersion horizontale (fig. 2). Elle consiste à comparer les caractéristiques statistiques (le déplacement quadratique 
moyen, ...) de la répartition de plusieurs bouées lâchées et celles obtenues grâce à des simulations numériques basées sur le champ eulérien observé et sur une représentation de la diffusion par une méthode de marche au hasard.

Après une brève présentation des mesures radar $(\& 2)$, on expose les principaux points de la méthode proposée (\& 3), puis on développe sur un exemple théorique une étude de faisabilité $(\& 4)$ et on discute les performances que l'on peut attendre, en rapport avec la précision des mesures, et la résolution du maillage.

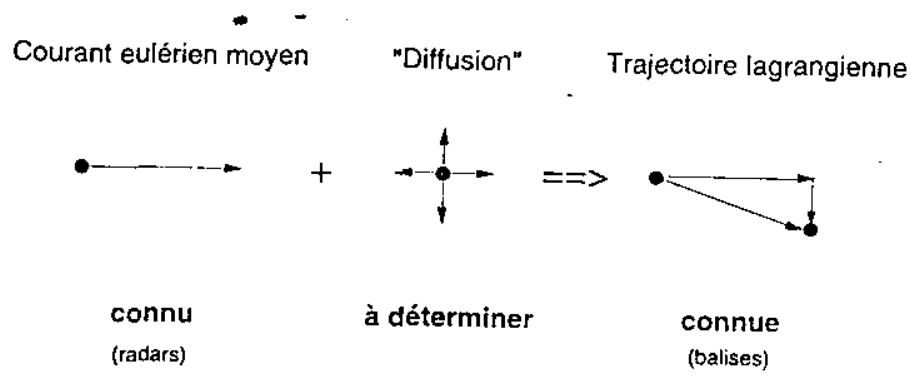

Figure 2 Schéma du problème étudié.

Illustration of the studied problem.

\section{2 - MESURE dES COURANTS PAR RADARS VHF}

La surface de la mer, agitée par des vagues, diffuse les ondes électromagnétiques et foumit un écho radar. Cette cible en mouvement produit un décalage Doppler sur l'écho dont l'analyse spectrale montre que l'essentiel de la puissance se trouve concentré dans deux raies quasimonochromatiques, " raies de Bragg", (dues à l'interaction résonnante entrè onde-radar et surface de la mer). Leur position est déterminée par la fréquence radar (CROMBIE, 1955 ; WAIT, 1966 ; BARRICK, 1972). Dans la pratique, le radar est placé au niveau de la mer (sur le rivage) et la propagation se fait principalement par onde de surface. Dans ces conditions d'incidence rasante, l'interaction "sélectionne" les vagues de longueur d'onde :

$$
\lambda_{B}=\frac{\lambda_{r}}{2}
$$

$\lambda_{r}$ étant la longueur d'onde radar.

D'après l'équation de dispersion des vagues libres, la vitesse de phase des vagues sélectionnées est donnée par :

$$
V_{B}=\sqrt{\frac{g \lambda_{r}}{4 \pi}}
$$


Les positions des deux raies de Bragg, en l'absence des courants marins, correspondent aux fréquences $\pm F_{B}, F_{B}$ étant la fréquence de Bragg, donnée par la formule générale de l'effet Doppler :

D'où finalement :

$$
F_{B}=\frac{2 V_{B}}{\lambda_{r}}
$$

$$
F_{B}(H z) \approx 0.102 \sqrt{t(M H z)}
$$

La présence d'un courantmakin superficiel crée une modification dans la vitesse des vagues. Cette variation de vitesse correspond, dans le domaine spectral, à un déplacement $\Delta$ fes raies par rapport à leur position théorique, déplacement donné par :

$$
\Delta f=\frac{\lambda_{r} U_{R}}{2}
$$

$\mathrm{U}_{\mathrm{R}}$ est la composante radiale de la vitesse de courant dans la direction d'observation (comptée négativement en s'éloignant du radar).

Le courant est mesuré à une profondeur de l'ordre de $\lambda_{r} / 8 \pi$ (STEWART et JOY, 1974).

Le temps nécessaire à la restitution d'une mesure de courant est typiquement de 6 minutes.

Pour obtenir une diversité spatiale, le fajsceau radar est pointé dans des directions contiguès et, pour chaque "cellule " de mer définie par un azimut et une distance, on calcule le spectre de puissance de l'écho, dont l'analyse foumit la composante radiale du courant dans cette cellule.

La réalisation d'une cartographie des courants marins superficiels nécessite l'utilisation de deux radars, installés sur des sites géographiques différents mais observant la même zone. Un exemple de mesures de courant par radar VHF sur le panache du Rhône (1994) est donné sur la figure 1.

\section{3 - ESTIMATION DU COEFFICIENT DE DIFFUSION HORIZONTALE}

La mesure de courant est intégrée sur une maille spatiale dont l'étendue est typiquement de $500 \times 500 \mathrm{~m}^{2}$. La variabilité du courant aux échelles inférieures à cet ordre de grandeur est lissée par la mesure. II en résulte que la trajectoire que l'on peut restituer à partir des cartes eulériennes n'est qu'une trajectoire "moyenne", dont les trajectoires "vraies " effectivement observées avec des bouées s'écarteront d'autant plus que la variabilité du courant à l'intérieur du domaine d'intégration est plus importante. Les fluctuations turbulentes inférieures à la maille d'acquisition radar sont paramétrées par un coefficient de diffusion qu'on cherche à estimer. On conçoit donc que la comparaison des positions mesurées avec la position 
moyenne permette d'estimer l'importance des phénomènes de diffusion turbulente.

On propose une méthode pour quantifier cette importance. Cette méthode est basée sur la comparaison des propriétés statistiques d'un ensemble de $N_{b}$ bouées avec les propriétés que l'on peut attendre en fonction du courant eulérien moyen et de l'importance de la diffusion, caractérisée par un coefficient constant $K_{h}$.

La prévision des propriétés statistiques s'appuie sur l'identification de la densité de probabilité de la position d'une bouée $P(x, y ; t)$ à la concentration d'un contaminant passif conservatif $C(x, y ; t)$ (normée par la condition de conservation, soit : $\left.\iint C(x, y ; t) d x d y=1 \forall t\right)$ et sur l'équation d'advectiondiffusion pour l'évolution de $P$ (BROOKS, 1960 ; FISCHER et al., 1979 ; LUHAR et al., 1993) :

$$
\frac{\partial P}{\partial t}+U \frac{\partial P}{\partial x}+V \frac{\partial P}{\partial y}=K_{h}\left(\frac{\partial^{2} P}{\partial x^{2}}+\frac{\partial^{2} P}{\partial y^{2}}\right)
$$

$(U, V)$ est le courant moyen (donc celui qui est fourni par les radars), $K_{h}$ est le coefficient de diffusion turbulente horizontale à déterminer. Connaissant la densité $P(x, y ; 0)$, cette équation fournit, pour chaque $K_{h}$, une densité $\hat{P}(x, y ; t)$.

La comparaison de $\hat{P}$ et $\tilde{P}$ permet d'estimer $K_{h}$. II s'agit en quelque sorte d'assimiler les valeurs observées $\tilde{P}$ pour, à l'aide du modèle défini par l'équation (1), estimer le paramètre $K_{h}$.

La réduction de la description des phénomènes turbulents au seul coefficient $K_{h}$ est certainement une simplification importante. Elle est néanmoins utilisée couramment par les auteurs qui s'intéressent à l'évolution des nappes superficielles (de pétrole, par exemple: e.g. ELLIOTT, 1986 ; ELLIOTT et al., 1989 ; MURRAY, 1972 ; LEHR et al., 1984 ; FAY, 1969). C'est ce qui a orienté notre choix. D'autre part, le coefficient obtenu est caractéristique des flotteurs utilisés. Leur petite taille est un argument pour supposer qu'il représente convenablement la turbulence de l'eau.

II faut noter que le coefficient de diffusion est généralement connu avec une marge d'erreur assez importante (souvent non précisée par les auteurs) : pour des environnements ayant des propriétés physiques, climatiques et hydrodynamiques proches, on observe dans la littérature une grande disparité concemant les valeurs du coefficient de diffusion.

Dans la pratique, on dispose simultanément :

- d'une estimation approchée de la vitesse du courant observé par radars sur une grille spatio-temporelle discrète (donc une réalisation bruitée),

- d'une estimation de la position du centre et de l'étendue d'une tache de diffusion obtenue par l'observation des positions d'un nombre suffisant de bouées.

La méthode est la suivante: avec le courant disponible, on simule l'effet de la diffusion par une méthode de la marche au hasard pour différentes valeurs du coefficient de diffusion horizontale $\left(K_{h}\right)$, et on établit des lois empiriques reliant les variances $\left(V_{X}, V_{Y}\right)$ (dans le repère des axes principaux: covariance nulle) au coefficient de diffusion $\left(K_{h}\right)$. Pour un coefficient de 
diffusion donné, on sera donc amené à construire les trajectoires d'un grand nombre de particules dont les évolutions sont le résultat combiné du courant et de la dispersion hydrodynamique due à la turbulence :

$$
V x=f\left(K_{h}\right) \text { et } V_{Y}=g\left(K_{h}\right)
$$

Si $S_{X}$ et $S_{Y}$ sont les estimations de la variance des positions, obtenues de l'observation de $N_{b}$ bouées à l'instant $t_{n}$, on en déduit une estimation de $K_{n}$.

\section{4 - MISE EN cEUVRE de LA MÉthode}

\subsection{Introduction}

En l'absence de données expérimentales, la méthode est illustrée par une expérience numérique. Celle-ci est fondée sur un modèle analytique de courant, développé par TAKANO (cité dans Defant, 1961) pour les panaches fluviaux et dans lequel la diminution rapide du courant par divergence à partir de l'embouchure est partiellement compensée par la diminution de l'épaisseur de la nappe d'eau en mouvement. On dispose ainsi d'un champ de courant qui présente quelque analogie avec celui que l'on rencontre sur le site de l'embouchure du Rhône (France), site test pour le LSEET.

Ce champ de courant est utilisé pour simuler :

- un champ eulérien analogue à celui que fournirait le système de deux radars VHF, c'est à dire des vitesses affectées d'une incertitude de mesure de quelques $\mathrm{cm} / \mathrm{s}$.

- la position d'un nombre $\mathrm{N}_{b}$ de flotteurs dérivants dans l'hypothèse d'un coefficient de diffusion donné.

Ces « données " sont ensuite mises en œuvre pour estimer un coefficient de diffusion, $\tilde{\mathrm{K}}_{\mathrm{h}}$, soit :

- calcul des propriétés statistiques (variances) de l'ensemble des $N_{b}$ flotteurs " observés".

- calcul, par la méthode de marche au hasard, des propriétés statistiques de la tache de diffusion évoluant sur le courant eulérien « mesuré ".

- évaluation de $\bar{K}_{h}$ par rapprochement des résultats précédents.

L'influence de la résolution spatiale et de la précision limitée des mesures eulériennes est discutée, ainsi que l'influence du nombre $N_{b}$ des bouées.

\subsection{Description du modèle de courant}

Les composantes du transport sont définies par (TAKANO, 1961):

$$
\left\{\begin{array}{l}
\bar{u}(x, y)=\frac{F_{0}}{2 \pi L}\left(\operatorname{Arctan}\left(\frac{y+L}{x}\right)-\operatorname{Arctan}\left(\frac{y-L}{x}\right)+\frac{2 L x\left(x^{2}-y^{2}+L^{2}\right)}{\left(x^{2}+(y+L)^{2}\left(x^{2}+(y-L)^{2}\right)\right.}\right) \\
\bar{v}(x, y)=\frac{F_{0}}{2 \pi L}\left(\frac{4 L x^{2} y}{\left(x^{2}+(y+L)^{2}\right)\left(x^{2}+(y-L)^{2}\right)}\right)
\end{array}\right.
$$


$F_{0}$ est le débit en volume du fleuve $\left(=1000 \mathrm{~m}^{3} / \mathrm{S}\right)$ et $2 \mathrm{~L}$ la largeur de l'embouchure $(=1000 \mathrm{~m})$.

Les composantes de la vitesse moyenne se déduisent des composantes du transport $(\bar{u}, \bar{v})$ et de l'épaisseur $d$ de la couche fluviale (approximative) grâce aux relations :

$$
\text { - }-\left\{\begin{array}{l}
U(x, y)=\frac{\bar{u}(x, y)}{d(x, y)} \\
V(x, y)=\frac{\bar{v}(x, y)}{d(x, y)}
\end{array}\right.
$$

Pour un profil exponentiel de la densité verticale du panache, l'épaisseur d est liée à la pression intégrée $Q$ par la relation suivante :

$$
d(x, y)=\sqrt{\frac{2 Q(x, y)}{5 g\left(\rho_{0}-\rho_{1}\right)}}
$$

avec :

$$
\begin{aligned}
Q(x, y) & =-\frac{F_{0} f}{2 \pi L}\left\{(y+L) \operatorname{Arctan}\left(\frac{y+L}{x}\right)-(y-L) \operatorname{Arctan}\left(\frac{y-L}{x}\right)\right\} \\
& +\frac{2 F_{0} A_{h}}{\pi}\left\{\frac{\left(x^{2}-y^{2}+L^{2}\right)}{\left(x^{2}+(y+L)^{2}\right)\left(x^{2}+(y-L)^{2}\right)}\right\}
\end{aligned}
$$

f est le coefficient de coriolis $\left(=10^{-4} \mathrm{~s}^{-1}\right), \rho_{1}$ la densité à la surface du panache ( $=1012 \mathrm{~kg} / \mathrm{m}^{3}$, salinité de $\left.15 \%\right), \rho_{0}$ la densité de l'eau marine $(=1030 \mathrm{~kg} /$ $\mathrm{m}^{3}$, salinité de $38 \%$ ) et $A_{h}$ est un coefficient caractéristique du modèle. Pour obtenir des vitesses de l'ordre de quelques dizaines de $\mathrm{cm} / \mathrm{s}$ (ordre de grandeur observé sur le terrain), nous avons choisi $A_{h}=8.10^{6} \mathrm{~m}^{2} / \mathrm{s}$. La Figure 3 présente les isolignes de la fonction de courant.

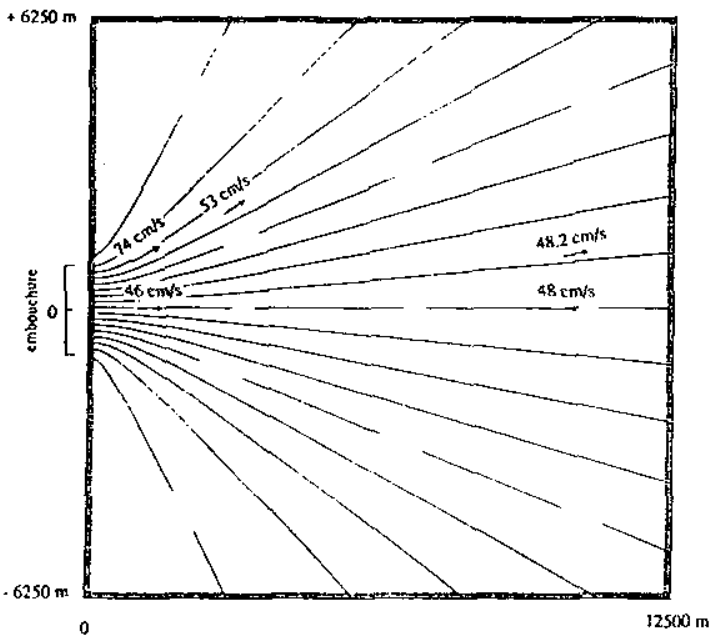

Figure 3 Isolignes de la fonction de courant (modèle de Takano) pour un débit en volume du fleuve de $1000 \mathrm{~m}^{3} / \mathrm{s}$ et une largeur de l'embouchure de $1000 \mathrm{~m}$ Stream function (Takano's model) for an outflow of $1000 \mathrm{~m}^{3} / \mathrm{s}$ and a width of the river mouth of $1000 \mathrm{~m}$. 
La grille utilisée est carrée avec un maillage régulier de quelques centaines de mètres (fig. 4). Les quantités $U$ et $V$ sont évaluées pour chaque nœud de la grille, défini par les coordonnées $\left(x_{i}, y_{i}\right)$, grâce aux expressions analytiques du vecteur courant.

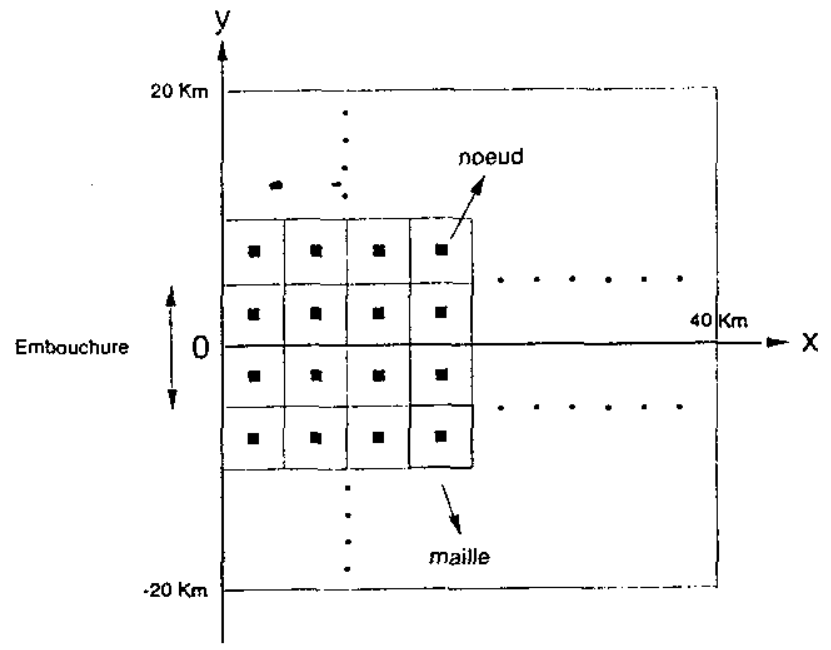

Figure 4 Discrétisation avec un maillage régulier.

Grid used with a regular size.

\subsection{Solution de l'équation de transport}

Il s'agit de définir une méthode pour résoudre, dans un champ d'advection $(\mathrm{U}, \mathrm{V})$ quelconque, l'équation (1). L'étude présentée suppose l'existence d'une diffusion "fickienne " avec un coefficient constant. C'est un problème classique, pour lequel est souvent proposée la méthode de la marche au hasard (AHLSTROM et FOOTE, 1976 ; ALLEN, 1993 ; ELLIOTT, 1986 ; ELLIOTT et al., 1989 ; GANOULIS, 1991-1992; HEEMINK, 1990 ; KINZELBACH et al., 1986 ; KINZELBACH, 1987 ; LECLERC et al., 1993 ; PRICKETT et al., 1981). Ce procédé de calcul considère la diffusion-dispersion comme un phénomène aléatoire. Le déplacement d'une particule peut être décrit par deux mouvements indépendants : le premier résulte du courant moyen et le second est généré par la dispersion des particules autour de leur position moyenne, mouvement aléatoire résultant de la turbulence au sein de l'écoulement.

La position d'une particule à l'instant $t_{n}(=n \Delta t)$ est calculée selon l'expression de récurrence :

$$
\left\{\begin{array}{l}
x_{n}=x_{n-1}+U_{n-1} \Delta t+\Delta x_{n} \\
y_{n}=y_{n-1}+V_{n-1} \Delta t+\Delta y_{n}
\end{array}\right.
$$

où $\left(U_{n-1}, V_{n-1}\right)$ est le vecteur courant au point où est située la particule à l'instant $t_{n-1}$ et $\Delta x_{n}, \Delta y_{n}$ deux nombres aléatoires qui prennent, avec des probabilités égales $(1 / 2)$, les valeurs $+\Delta x$ et $+\Delta y$ respectivement, avec : 


$$
\Delta x=\Delta y=\sqrt{2 K_{h} \Delta t}
$$

La moyenne des $\Delta x_{n}$ et des $\Delta y_{n}$ est nulle et leur variance est de $\left(2 K_{h} \Delta t\right)$.

Le pas de temps $\Delta t$ est choisi de manière que $\Delta x$ et $\Delta y$ soient bien inférieurs à $U \Delta t$ et $V \Delta t$ respectivement et pour que chaque déplacement de la particule ne dépasse pas la moitié du pas de maillage (GANOULIS, 1992; ELLIOTT, 1986 ; ELLIOTT et al., 1989).

Pour simuler les trajectoires des bouées lâchées pendant une expérience, on utilise l'algorithme ci-dessus en prenant pour courant le courant "vrai ", fourni par le champ analytique en chaque point (le courant n'est donc pas affecté d'erreurs).

Pour résoudre l'équation d'advection-diffusion, on utilise le même algorithme en prenant comme courant en chaque point le résultat d'une interpolation qui associe les valeurs (affectées d'une incertitude) aux quatre nœuds voisins de la maille.

\section{5 - PERFORMANCES DE LA MÉTHOdE - ÉVALUATION THÉORIQUE}

\subsection{Sans diffusion}

On calcule les éléments de la trajectoire d'une particule dans le champ eulérien décrit dans le paragraphe 3.3 , en négligeant pour l'instant les effets de la diffusion.

En l'absence de toute imprécision, ces trajectoires coïncident avec les lignes de courant. Dans la pratique, leur calcul s'effectue à partir de mesures bruitées, et nécessite des interpolations. Ces deux aspects sont des causes d'erreur que l'on étudie ci-dessous.

Le schéma numérique utilisé pour le calcul de ces trajectoires est le schéma simple de différences finies:

$$
\left\{\begin{array}{l}
x_{i+1}=x_{i}+U_{i} \Delta t \\
y_{i+1}=y_{i}+V_{i} \Delta t
\end{array}\right.
$$

avec $\left(U_{i}, V_{i}\right)$ le vecteur courant au point $\left(x_{i}, y_{i}\right)$, obtenu par une interpolation linéaire sur les quatre nœuds qui l'entourent et $\Delta t$ le pas de temps.

Si chaque vitesse utilisée est affectée d'une incertitude $(\delta \mathrm{U}, \delta \mathrm{V})$ caractérisant le bruit de mesure lié aux instruments et l'erreur induite par l'interpolation du vecteur courant, le schéma de calcul devient :

$$
\left\{\begin{array}{l}
\tilde{x}_{i+1}=\tilde{x}_{i}+\left(\tilde{U}_{i}+\delta \tilde{U}_{i}\right) \Delta t \\
\tilde{y}_{i+1}=\bar{y}_{i}+\left(\bar{V}_{i}+\delta \tilde{V}_{i}\right) \Delta t
\end{array}\right.
$$


avec:

$$
\left(\tilde{U}_{i}, \tilde{V}_{i}\right)=\left(U\left(\tilde{x}_{i}, \tilde{y}_{i}\right), V\left(\tilde{x}_{i}, \tilde{y}_{i}\right)\right) \text { et }\left(\delta \tilde{U}_{i}, \delta \tilde{V}_{i}\right) \quad \text { l'incertitude correspondante. }
$$

En faisant localement l'hypothèse de linéarité pour les variations spatiales du courant :

$$
\left\{\begin{array}{l}
\tilde{U}_{i}=U_{i}+\left.\left(\tilde{x}_{i}-x_{i}\right) \frac{\partial U}{\partial x}\right|_{x_{i}} \\
\tilde{y}_{i}=V_{i}+\left.\left(\bar{y}_{i}-y_{i}\right) \frac{\partial V}{\partial y}\right|_{y_{i}}
\end{array}\right.
$$

avec:

on peut écrire :

$$
\xi_{i}^{x}=\left(\tilde{x}_{i}-x_{i}\right), \xi_{i}^{y}=\left(\tilde{y}_{i}-y_{i}\right), \alpha_{i}^{x}=\left.\frac{\partial U}{\partial y}\right|_{y_{i}}
$$

$$
\begin{aligned}
\xi_{n}^{x}= & \left(1+\alpha_{n-1}^{x} \Delta t\right) \ldots\left(1+\alpha_{1}^{x} \Delta t\right) \delta \tilde{U}_{0} \Delta t+\left(1+\alpha_{n-1}^{x} \Delta y\right) \ldots\left(1+\alpha_{2}^{x} \Delta t\right) \delta U_{1} \Delta t \\
& +\ldots+\left(1+\alpha_{n-1}^{x} \Delta t\right) \delta \tilde{U}_{n-2} \Delta t+\delta \tilde{U}_{n-1} \Delta t
\end{aligned}
$$

$\xi_{n}^{y}$ est donné par une formule analogue : il suffit de remplacer $\alpha^{x}$ par $\alpha^{y}$ et $\delta \tilde{U} \operatorname{par} \delta \tilde{V}$.

$\xi_{n}^{x}$ et $\xi_{n}^{y}$ sont les sommes de $n$ variables gaussiennes indépendantes (proportionnelles à $\delta \tilde{U}_{i}$ par $\delta \tilde{V}_{i}$ respectivement). Elles suivent donc des lois normales de moyennes:

$$
<\xi_{n}^{x, y}>=0
$$

et de variances :

$$
\begin{aligned}
\operatorname{Var}\left(\xi_{n}^{x, y}\right)= & \left\{1+\left(1+\alpha_{n-1}^{x, y} \Delta t\right)^{2}+\left(1+\alpha_{n-1}^{x, y} \Delta t\right)^{2}\left(1+\alpha_{n-2}^{x, y} \Delta t\right)^{2}\right. \\
& \left.+\ldots+\left(1+\alpha_{n-1}^{x, y} \Delta t\right)^{2} \ldots\left(1+\alpha_{1}^{x, y} \Delta t\right)^{2}\right\} \sigma_{U, V}^{2} \Delta t^{2} \\
& =\beta_{n}^{x, y} \sigma_{U, V}^{2} \Delta t^{2}
\end{aligned}
$$

si les incertitudes $\delta \tilde{U}_{i}$ (resp. $\delta \tilde{V}_{i}$ ) sont indépendantes et de même variance $\sigma_{U}^{2}$ (resp. $\sigma_{V}^{2}$ ).

Le premier résultat montre que la procédure de trajectographie n'est pas biaisée : si l'on pouvait disposer d'un très grand nombre de " réalisations expérimentales", la position moyenne obtenue serait très voisine de la position vraie.

Le second résultat donne une indication sur la précision d'une reconstitution. Elle dépend de l'erreur expérimentale (erreur d'interpolation incluse) et de la structure du champ de courant (coefficients $\alpha_{i}^{x_{1} y} \Delta t$ ). 


\subsection{En présence de diffusion}

Il a été exposé (cf. \& 4.3) que l'on peut simuler les processus de diffusion par une méthode stochastique. Pour un coefficient de diffusion donné, on est amené à construire les trajectoires d'un grand nombre de particules régies par:

$$
\left\{\begin{array}{l}
\tilde{x}_{i+1}=\tilde{x}_{i}+\tilde{U}_{i} \Delta t+\Delta \tilde{x}_{i+1} \\
\tilde{y}_{i+1}=\tilde{y}_{i}+\tilde{V}_{i} \Delta \tilde{y}_{i+1}
\end{array}\right.
$$

$(\tilde{U}, \tilde{V})$ est le courant « bruité " résultant des mesures, et $(\Delta x, \Delta y)$ le saut aléatoire associé au processus de diffusion. Si l'on compare à la trajectoire « vraie " obtenue en l'absence de diffusion et définie par $\left\{x_{i+1}, y_{i+1}\right\}$ (éq. (3)), on trouve (avec l'hypothèse de linéarité locale):

$$
\left\{\begin{array}{l}
\xi_{n}^{x}=\left(\tilde{x}_{n}-x_{n}\right)=\left(1+\alpha_{n-1}^{x} \Delta t\right) \xi_{n-1}^{x}+\delta \tilde{U}_{n-1} \Delta t+\Delta \tilde{x}_{n} \\
\xi_{n}^{y}=\left(\tilde{y}_{n}-y_{n}\right)=\left(1+\alpha_{n-1}^{y} \Delta t\right) \xi_{n-1}^{y}+\delta \tilde{V}_{n-1} \Delta t+\Delta \tilde{y}_{n}
\end{array}\right.
$$

$\alpha_{i}^{x}$ et $\alpha_{i}^{y}$ sont définis dans le paragraphe 5.1.

Soit finalement :

$$
\begin{aligned}
\xi_{n}^{x}= & \left(1+\alpha_{n-1}^{x} \Delta t\right) \ldots\left(1+\alpha_{1}^{x} \Delta t\right)\left(\delta \tilde{U}_{0} \Delta t+\Delta \tilde{x}_{1}\right)+\left(1+\alpha_{n-1}^{x} \Delta t\right) \ldots\left(1+\alpha_{2}^{x} \Delta t\right)\left(\delta \tilde{U}_{1} \Delta t+\Delta \bar{x}_{2}\right) \\
& +\ldots+\left(1+\alpha_{n-1}^{x} \Delta t\right)\left(\delta \tilde{U}_{n-2} \Delta t+\Delta \tilde{x}_{n-1}\right)+\left(\delta \tilde{U}_{n-1} \Delta t+\Delta \tilde{x}_{n}\right) \\
\xi_{n}^{y} & =\left(1+\alpha_{n-1}^{y} \Delta t\right) \ldots\left(1+\alpha_{1}^{y} \Delta t\right)\left(\delta \tilde{V}_{0} \Delta t+\Delta \tilde{y}_{1}\right)+\left(1+\alpha_{n-1}^{y} \Delta t\right) \ldots\left(1+\alpha_{2}^{y} \Delta t\right)\left(\delta \tilde{V}_{1} \Delta t+\Delta \tilde{y}_{2}\right) \\
& +\ldots+\left(1+\alpha_{n-1}^{y} \Delta t\right)\left(\delta \tilde{V}_{n-2} \Delta t+\Delta \tilde{y}_{n-1}\right)+\left(\delta \tilde{V}_{n-1} \Delta t+\Delta \tilde{y}_{n}\right)
\end{aligned}
$$

Pour un très grand nombre de particules et une réalisation unique de courant, on trouve:

$$
\begin{aligned}
& \left.<\xi_{n}^{x}\right\rangle=\left(1+\alpha_{n-1}^{x} \Delta t\right) \ldots\left(1+\alpha_{1}^{x} \Delta t\right) \delta \tilde{U}_{0} \Delta t+\left(1+\alpha_{n-1}^{x} \Delta t\right) \ldots\left(1+\alpha_{2}^{x} \Delta t\right) \delta \tilde{U}_{1} \Delta t+\ldots+\delta \tilde{U}_{n-1} \Delta t \\
& \left.<\xi_{n}^{y}\right\rangle=\left(1+\alpha_{n-1}^{y} \Delta t\right) \ldots\left(1+\alpha_{1}^{y} \Delta t\right) \delta \tilde{V}_{0} \Delta t+\left(1+\alpha_{n-1}^{y} \Delta t\right) \ldots\left(1+\alpha_{2}^{y} \Delta t\right) \delta \tilde{V}_{1} \Delta t+\ldots+\delta \tilde{V}_{n-1} \Delta t
\end{aligned}
$$

II y a donc un biais qui est dû aux incertitudes sur la réalisation unique associée à une observation du courant.

Nous avons simulé à partir d'une seule réalisation du champ radar (champ eulérien) et pour différentes valeurs de $K_{h}$ le barycentre de 1000 particules (fig. 5). Nous constatons un biais entre les barycentres et la position « vraie " (celle obtenue si les vecteurs courant sont calculés directement à partir du champ analytique de courant) ; ceci est associé à la réalisation unique du champ de courant. 


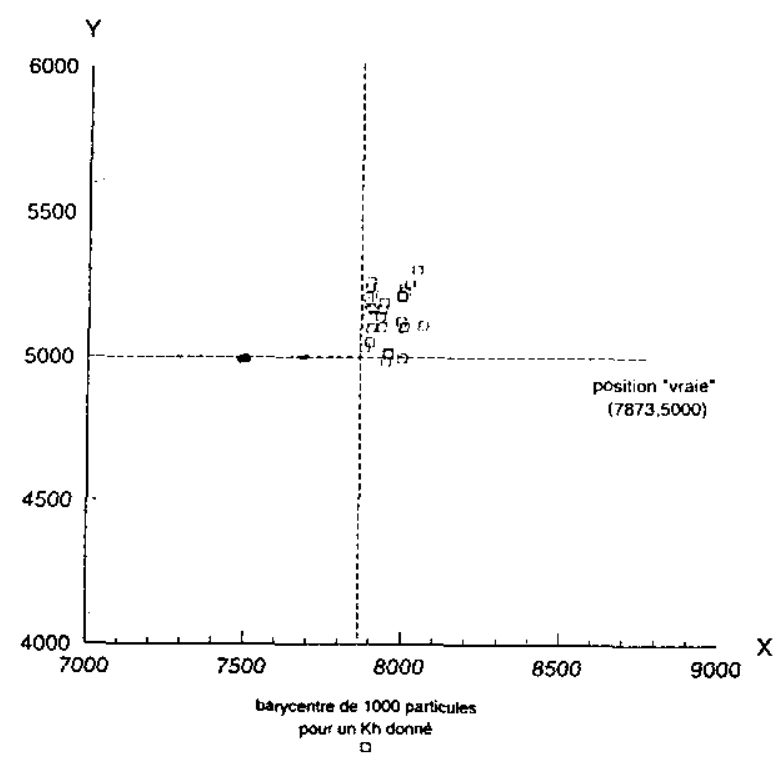

Figure 5 Barycentre d'un nuage de 1000 particules simulé à partir d'une réalisation du champ radar (simulée grâce au modẻle de Takano) pour différentes valeurs du coefficient de diffusion.

Mean position of 1000 particles after a simulation of their trajectories over a periode of 4 hours using for several diffusion coefficient and a current field supplied by Takano's model.

On montre que la variance s'écrit comme suit :

$$
\begin{aligned}
\operatorname{Var}\left(\xi_{n}^{x, y}\right) & =\left\{1+\left(1+\alpha_{n-1}^{x, y} \Delta t\right)^{2}+\left(1+\alpha_{n-1}^{x, y} \Delta t\right)^{2}\left(1+\alpha_{n-2}^{x, y} \Delta t\right)^{2}\right. \\
& \left.+\ldots+\left(1+\alpha_{n-1}^{x, y} \Delta t\right)^{2} \ldots\left(1+\alpha_{1}^{x, y} \Delta t\right)^{2}\right\}(\Delta x, y)^{2} \\
& =\beta_{n}^{x, y}(\Delta x, y)^{2} \\
& =\left(2 K_{n} \Delta t\right) \beta_{n}^{x, y}
\end{aligned}
$$

Celle-ci n'est pas affectée par le biais : autrement dit, la « tache de diffusion " reconstituée est centrée en un point erroné, mais sa dimension est identique à celle que l'on aurait obtenue avec le courant "vrai ".

La position reconstituée est centrée sur $\left(\left\langle\xi_{n}^{x}\right\rangle,\left\langle\xi_{n}^{y}\right\rangle\right)$, avec une densité de probabilité gaussienne de variance $\left\{\operatorname{Var}\left(\xi_{n}^{x}\right), \operatorname{Var}\left(\xi_{n}^{y}\right)\right\}$.

D'autre part, la valeur moyenne $\overline{\left\langle\xi_{n}^{x, y}\right\rangle}$ et la variance $\left\{\overline{\left.\left\langle\xi_{n}^{x, y}\right\rangle-\overline{<\xi_{n}^{x, y}}\right\rangle}\right\}^{2}$ du biais sont égales à 0 et à $\left(\beta_{n}^{x, y} \sigma_{U, V}^{2} \Delta t^{2}\right)$ respectivement (pour un grand nombre de réalisations de courant). 
Les calcuis précédents ont montré que dans le cadre de l'hypothèse de linéarité locale, on a :

$$
\left\{\begin{array}{l}
V_{X}=\left(2 \beta_{n}^{x} \Delta t\right) K_{h}+\beta_{n}^{x} \sigma_{U}^{2} \Delta t^{2} \equiv Q_{n} K_{h}+L_{n} \\
V_{Y}=\left(2 \beta_{n}^{y} \Delta t\right) K_{h}+\beta_{n}^{y} \sigma_{V}^{2} \Delta t^{2} \equiv P_{n} K_{h}+Z_{n}
\end{array}\right.
$$

$L_{n}$ et $Z_{n}$ sont des résidus puisqu'ils contiennent essentiellement les erreurs de la mesure des courants eulériens. On n'en tient pas compte dans l'estimation du coefficient_de diffusion.

Pour déterminer le coefficient de diffusion $\left(K_{h}\right.$, on inverse les relations (5) et on utilise la méthode des moindres carrés (BRANDT, 1970 ; MENKE, 1984). Le meilleur estimé de $\mathrm{K}_{h}$ est donc :

$$
\tilde{K}_{h}=\frac{Q_{n} S_{X}+P_{n} S_{Y}}{P_{n}^{2}+Q_{n}^{2}}
$$

\section{6 - RÉSULTATS ET DISCUSSION}

L'incertitude sur les mesures du courant eulérien est assimilable à un bruit gaussien de moyenne nulle et d'écart type $5 \mathrm{~cm} / \mathrm{s}$ (dans la pratique, la précision sur la mesure du courant par radars est de cet ordre de grandeur) : pour simuler un champ de courant radar, on discrétise le champ analytique de courant sur une grille spatiale afin d'obtenir le vecteur vitesse en tout nœud. On ajoute ensuite aux vecteurs vitesses un bruit gaussien de moyenne nulle et d'écart type $5 \mathrm{~cm} / \mathrm{s}$ pour leur intégrer le bruit de mesure.

La reconstitution des trajectoires lagrangiennes à partir des données eulériennes de courant est obtenue grâce à l'équation (4) : pour différentes valeurs de $\mathrm{K}_{\mathrm{hi}}$ et un champ eulérien bruité puis interpolé, on simule les trajectoires d'un grand nombre de particules (1000) et on calcule après un temps total de dérive de 4 heures les variances $V_{X}\left(K_{h i}\right)$ et $V_{Y}\left(K_{h_{i}}\right)$ Ensuite, on établit des lois empiriques reliant les variances au coefficient de diffusion par les relations linéaires définies par (5).

Les pas de maillage choisis sont de 100,300 et $500 \mathrm{~m}$ avec des pas de temps $\Delta t$ de 1,3 et 5 minutes respectivement. Les valeurs utilisées du coefficient de diffusion appartiennent à l'intervalle $[0.01,1] \mathrm{m}^{2} / \mathrm{s}$, intervalle contenant tous les ordres de grandeurs rencontrées dans la littérature (DAVIS, 1985 ; ELLIOTT, 1986 ; ELLIOTT et al., 1989 ; HAYNES et al., 1991 ; OKUBO, 1967-1971).

Dans le but d'obtenir une estimation fiable de la précision sur $K_{h}$, on considère un nombre suffisamment élevé de réalisations du champ de courant eulérien afin d'établir les intervalles de confiance à $95 \%$ des lois reliant $V_{X}$ et $V_{Y}$ à $K_{h}$ (BENDAT et PIERSOL, 1971). 
Les intervalles de confiance à $95 \%$, pour 1000 degrés de liberté (1001 champs eulériens), des variances $V_{X}=f\left(K_{h}\right)$ et $V_{Y}=g\left(K_{h}\right)$ sont de la forme:

$$
\left\{\begin{array}{l}
<V_{X}>_{95 \%}=<Q_{n}>_{95 \%} K_{h}+<L_{n}>_{95 \%} \\
<V_{Y}>_{95 \%}=<P_{n}>_{95 \%} K_{h}+<Z_{n}>_{95 \%}
\end{array}\right.
$$

Dans la pratique, on dispose d'une réalisation unique du champ de courant eulérien.

Le point de départ des pärticles (point de lâcher) est $x=1500 \mathrm{~m}$ et $\mathrm{y}=$ $1000 \mathrm{~m}$.

La dérive de $N_{b}$ bouées est également simulée durant 4 heures (éq. (2)). Avant de calculer l'étendue de la tache de diffusion $\left(S_{X}\left(K_{h i}\right), S_{Y}\left(K_{h i}\right)\right)$ dans le repère de covariance nulle, on bruite les mesures de positions, par un bruit gaussien de moyenne nulle et d'écart type $100 \mathrm{~m}$ afin d'introduire l'incertitude sur leurs localisations.

Le nombre de bouées $\mathrm{N}_{b}$ utilisé dans les simulations est de $5,10,20,30$, 40 et 50 . Le pas de temps est d'une minute (pour restituer au mieux une trajectoire lagrangienne vraie, il faut utiliser un pas de temps très petit et par conséquent, un pas d'une minute s'est révélé suffisant).

Les lâchers de $N_{b}$ bouées sont répétés une centaine de fois afin d'établir, pour chaque coefficient $K_{h i}$, les intervalies de confiance à $95 \%$ de $S_{X}$ (Khi) et de $S_{\mathrm{Y}}\left(K_{\mathrm{hi}}\right)$.

Il faut bien noter que dans la pratique, $S_{X}$ et $S_{Y}$ sont obtenues grâce à un lâcher unique de $N_{b}$ bouées.

La Figure 6 représente l'erreur relative sur l'estimation du coefficient de diffusion en fonction du nombre de particules (ou de bouées) après une durée $t_{n}$ de 4 heures et pour un pas de maillage de 100,300 et $500 \mathrm{~m}$ respectivement. II s'agit de la borne supérieure et de la borne inférieure de l'erreur ainsi que de leur moyenne.

On en déduit les conclusions suivantes:

- l'erreur relative sur l'estimation de $K_{h}$ diminue quand le nombre de bouées augmente.

- l'erreur relative sur l'estimation de $K_{h}$ diminue quand la dimension des mailles de la grille d'acquisition radar diminue.

- pour un pas de maillage quelconque et un nombre de bouées lâchées supérieur ou égal à 10 , l'erreur relative moyenne sur l'estimation du coefficient de diffusion est quasiment identique : elle est de l'ordre de $10 \%$ pour un pas de maillage de $100 \mathrm{~m}$ et de $20 \%$ pour un pas de $500 \mathrm{~m}$.

- pour un lâcher de 5 bouées et un pas de maillage de $500 \mathrm{~m}$ (ordre de grandeur réaliste de la dimension des mailles), l'erreur relative moyenne sur l'estimation de $\mathrm{K}_{h}$ est de $35 \% \pm 10 \%$.

On peut remarquer que la connaissance de la circulation ambiante moyenne grâce à des radars océaniques est décisive pour une bonne estimation du coefficient de diffusion. 

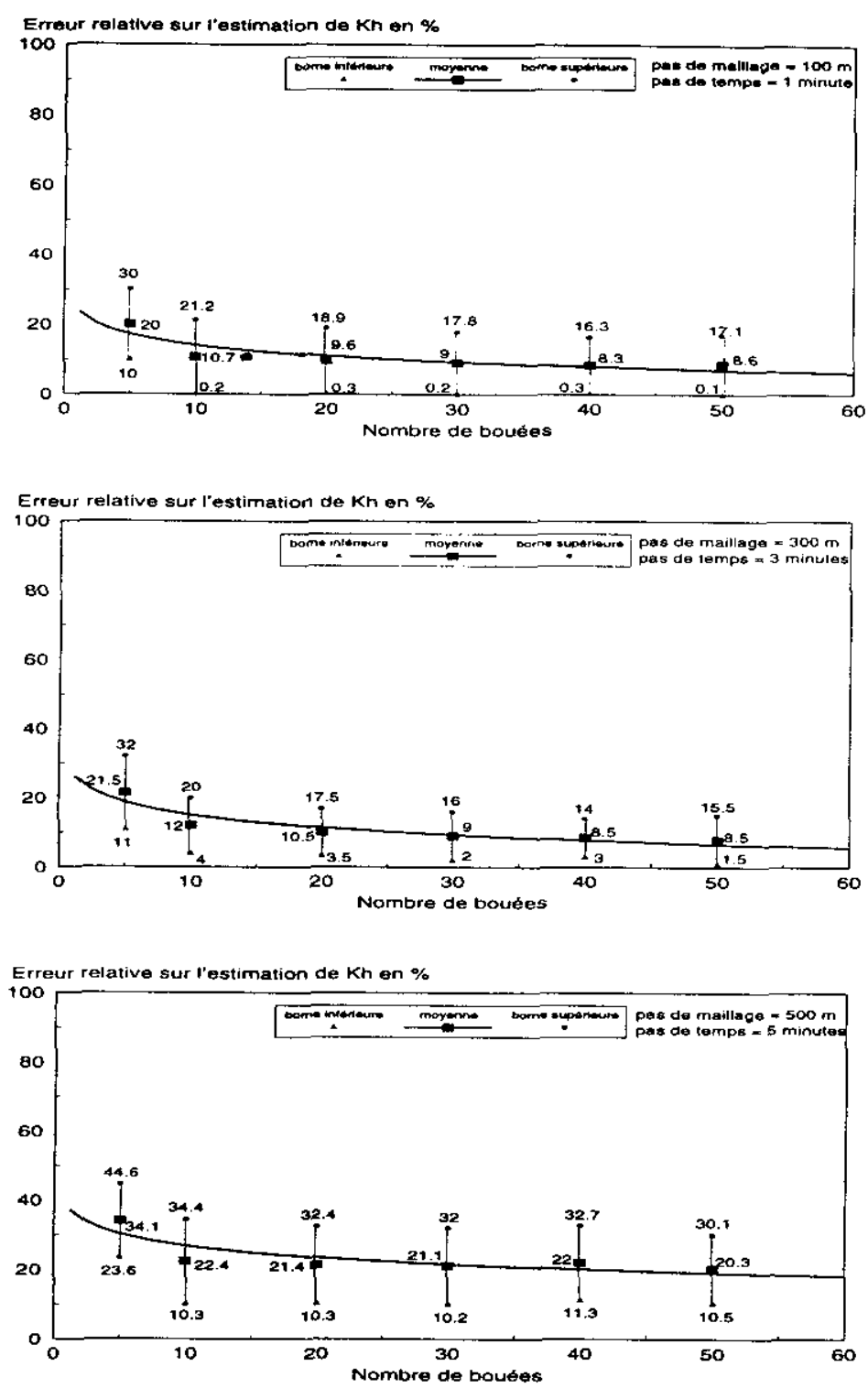

Figure 6 Erreur relative sur l'estimation du coefficient de diffusion horizontale en fonction du nombre de particules. Le pas de maillage est respectivement de 100,300 et $500 \mathrm{~m}$. La restitution des trajectoires lagrangiennes est effectuée sur une durée de 4 heures.

Relative error of the horizontal diffusion coefficient estimation as a function of the number of drifters. The spatial resolution of current measurements is of 100,300 and $500 \mathrm{~m}$. The duration of the numerical simulation is 4 hours. 
La Figure 7 représente les résultats d'une simulation numérique concernant le suivi de 1000 flotteurs durant 4 heures, d'une part avec un courant uniforme (courbe 1) sur toute la zone d'étude (ce que l'on ferait si on ne disposait que d'instruments ne possédant pas une grande couverture spatiale, les courantomètres par exemple; ou bien si la résolution spatiale de la mesure de courant est très grande et donc insuffisante pour des applications à l'échelle côtière) et d'autre part avec un courant analytique, celui de Takano, (courbe 2) fournissant un vecteur courant en tout point de la zone d'étude (pour simuler au mieux des données radars).

Pour une dispersion donnée, te coefficient de diffusion horizontale estimé grâce à la courbe (1) est bien différent de celui obtenu par la courbe (2) qui est supposé vrai. Le coefficient obtenu par (1) est un mélange de fluctuations turbulentes et du gradient de vitesse.

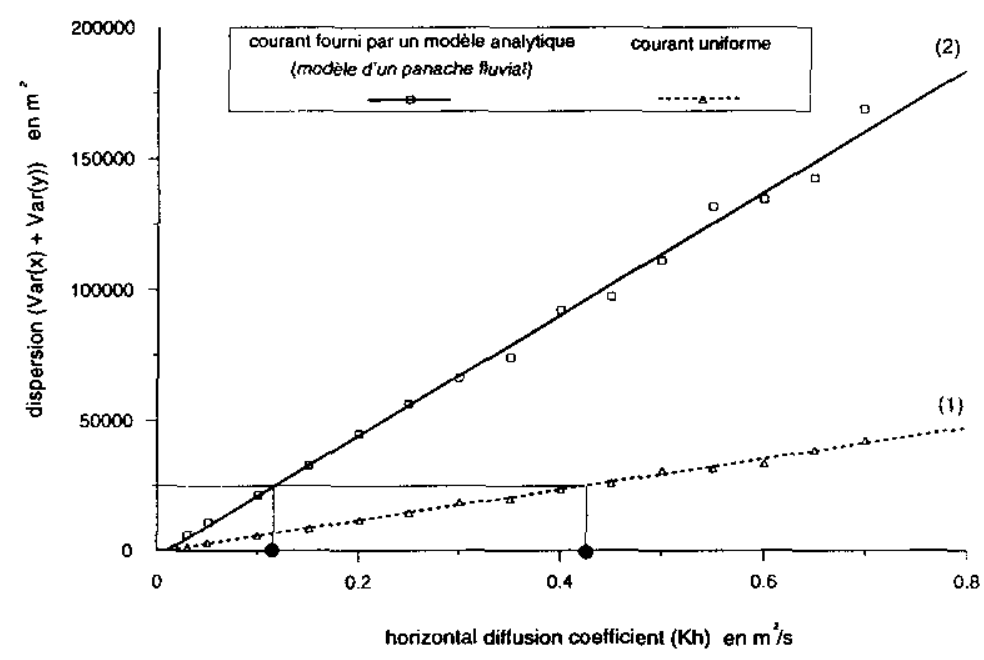

Figure 7 Résultats d'une simulation numérique concernant la dispersion d'un nombre suffisant de flotteurs $(1000)$ durant 4 heures. Pour un coefficient de diffusion $\left(K_{p}\right)$ donné et un champ de courant uniforme ou de type panache fluvial, la dispersion des flotteurs est calculée pour obtenir respectivement les courbes (1) et (2).

Numerical simulation results for the dispersion of a sufficient number of drifters (1000) over a period of 4 hours. For a given diffusion coefficient $\left(K_{h}\right)$ and for a uniform current field or a current field known at each point (model of river plume), the drifters' dispersion is computed in order to obtain the curves (1) and (2), respectively (the lines represent the best fits).

\section{7 - CONCLUSiON générale et PERSPECtives}

Nous avons montré sur un exemple qu'il est possible, par utilisation couplée de données eulériennes et lagrangiennes de courant, d'obtenir avec 
une précision convenable une estimation du coefficient de diffusion horizontale.

Nous avons étudié l'influence du pas de maillage et celui des erreurs de mesures du courant sur la restitution des trajectoires lagrangiennes. Deux conclusions ont été tirées de cette étude, la première est que la procédure de trajectographie ne serait pas biaisée si f'on pouvait disposer d'un très grand nombre de cartes de courant; la seconde montre que la précision sur la reconstitution d'une dérive lagrangienne dépend de l'erreur expérimentale (erreur d'interpolation incluse) et de la structure du champ de courant. Pour le modèle de panache, nous avons montré que l'étendue de la tache de diffusion est proportionnelle au coëfficíent de diffusion horizontale et que l'estimation de ce dernier, d'après les simulations numériques, est effectuée avec une erreur relative moyenne de l'ordre de $20 \%$ pour un lâcher de 10 bouées et un pas de maillage de $500 \mathrm{~m}$ et de l'ordre de $35 \%$ pour 5 particules lâchées et un pas de maillage de $500 \mathrm{~m}$. Nous avons également montré que la connaissance de la circulation générale moyenne grâce à des radars océaniques est importante pour une bonne estimation du coefficient de diffusion.

Cela est important pour la modélisation des phénomènes de transport en zones côtières, de grande importance pour les études d'environnement.

Le système (radars et bouées) est un instrument particulièrement complet de diagnostic dynamique en zone côtière, présentant un grand intérêt pour l'étude d'un rejet ou le transport d'un contaminant. II est susceptible d'apporter aux études de courantologie côtière une contribution originale. Par manque de données expérimentales, nous n'avons pas pu valider la méthode de calcul du coefficient de diffusion. Des résultats expérimentaux sont néces* saires pour confirmer le système radars-bouées comme un outil opérationnel d'étude des sites littoraux.

\section{RÉFÉRENCES BIBLIOGRAPHIQUES}

AHLSTROM S.W., FOOTE H.P., 1976. Transport modelling in the environment using the discrete-parcel-random-walk approach. Proc. of the Conf. Env. Modelling Simulation, Ott., EPA 60019. 76-016, pp. 833-837.

ALLEN C.M., 1993. Modelling contaminant dispersion in the River Severn using a random-walk model. Joumal of Hydraulic Research, vol. 31, no. 3, pp. 323-331.

BAGHDADI N., BROCHE P., GAGGELLI J., 1994. Courantométrie eulérienne et lagrangienne par radar VHF, 3emes journées nationales Génie civil-Génie côtier; SETE-FRANCE.
BARRICK D.E., 1972. Remote sensing of the sea state by radar. Remote Sensing of the Troposphere, V. DERR, Ed. Washington, DC : U.S. Government printing office, ch. 12, pp. 1-46.

BENDAT J.S., PIERSOL, 1971, Random data : analysis and measurement procedures. Edition Wiley and Sons, New York.

BRANDT S., 1970. Statistical and computational methods in data analysis. Amsterdam, North Holland, second revised edition, $414 \mathrm{pp}$.

BROCHE P., FORGET P., DE MAISTRE J.C., DEVENON J.L., CROCHET M., 1987. VHF radar for ocean surface current and sea 
state remote sensing. Radio Science, vol. $22, n^{\circ} 1$, pp. 69-75.

BROOKS N.H., 1960. Diffusion of sewage effluents in an ocean current. Waste disposal in the marine environment. Pergamon Press

CROMBIE D., 1955. Doppler spectrum of sea echo at $13.56 \mathrm{mc} . / \mathrm{s}$. Nature, vol. 175 , pp.681-682.

DAVIS R.E., 1985. Drifter obsermations of coastal surface currents during CODE : The statistical and dynamical views. Joumal of Geophysical Research, vol. 90, $\mathrm{n}^{\circ} \mathrm{C} 3$, pp. $4756-4772$.

DEVENON J.L., BROCHE P., DE MAISTRE J.C., FORGET P., GAGGELLI J., ROUGIER G., 1991. VHF radar measurements in the Rhône river plume. 3rd workshop of the EROS 2000 Project, Texel, Nederlands.

ELLIOTT A.J., 1986. Shear diffusion and the spread of oil in the surface layers of the North sea. Dt. Hydrogr. Z., 39, H.3., pp. 113-137.

ELLIOTT A.J., HURFORD N., 1989. The influence of wind and wave shear on the spreading of a plume at sea. Oil and Chemical Pollution, vol. 5, pp. 347-363.

FAY J.A., 1969. The spread of oil slicks on a calm sea. In: oil on the sea. New York. Plenum. pp. 53-63.

FISCHER H.H., LIST J., KOH R., IMBERGER J., BROOKS N.H., 1979. Mixing in inland an coastal waters. Academic Press.

GANOULIS J., 1991. Water quality assessment and protection measures of a semi-enclosed coastal area. Marine Pollution Bulletin, 23, 83-87.

GANOULIS J., 1992. Dispersion et disparition des bactéries coliformes dans la baie de Thessaloniki. Revue des Sciences de leau, 3, 541-554.

HAYNES R., BARTON E.D., 1991. Lagrangian observations in the Iberian coastal transition zone. Journal of Geophysical Research, $96, n^{\circ} \mathrm{C} 8,14,731-14,741$.

HEEMINK A.W., 1990. Stochastic modelling of dispersion in shallow water. Stoch. Hydrology and Hydraulics, 4, 161-174.
KINZELBACH W., ACKERER P., 1986. Modélisation de la propagation d'un contaminant dans un champ d'écoulement transitoire. Hydrogéologie, 2, 197-206.

KINZELBACH W., 1987. Methods for the simulation of pollutant transport in ground water - A model comparison. Proceeding of the solving ground water problems with models, conference and exposition, vol. 1 , Denver Colorado, pp. 656-675.

LECLERC M., BOUDREAULT P., 1993. Méthodologie d'analyse détaillée de la contamination par tronçon du fleuve SaintLaurent par modélisation numérique: le cas du lac Saint-Pierre. Revue des sciences de l'eau, 6, 4, 427-451.

LEHR W.J., CEKIRGE H.M., FRAGA R.J., BELEN M.S., 1984. Empirical studies of the spreading of oil spills. Oil \& Petrochem. Poll., 2, 7-11.

LUHAR A.K., RAO K.S., 1993. Random-walk model studies of the transport and diffusion of pollutants in katabatic flows. Boundary Layer Meteorology, 66, 395-412.

MENKE W., 1984. Geophysical data analysis : discrete inverse theory. Academic Press, Inc., $254 \mathrm{pp}$.

MURRAY S.P., 1972. Turbulent diffusion of oil in the ocean. Limnol. Oceanol., 17, 651660.

OKUBO A., 1967. The effect of shear in an oscillatory current on horizontal diffusion from an instantaneous source. Limnol. Oceanol., 1, 194-204.

OKUBO A., 1971. Ocean diffusion diagrams, Deep Sea Res., 18, 789-802.

PRICKETT T.A., NAYMIK T.G., LONNQUIST C.G., 1981. A random-walk solute transport model for selected ground water quality evaluations. Illinois state water survey, Champaign, Bulletin 65, 101 pp.

STEWART R.H., JOY J.W., 1974. HF radio measurements of surface currerts. Deep Sea Res., 21, 1039-1049.

TAKANO K., 1961. Extrait du livre de Albert Defant: Physical Oceanography, vol. 1, pp. 538-543. Pergamon Press Inc., New York.

WAIT J.R., 1966. Theory of H.F. ground wave backscatter from sea waves. Journal of Geophysical Research, 71, 4839-4855. 